\title{
Evolving Energy Demand Estimation Models over Macroeconomic Indicators
}

\author{
Nuno Lourenço \\ University of Coimbra, CISUC, DEI \\ Coimbra, Portugal \\ naml@dei.uc.pt \\ J. Ignacio Hidalgo \\ Universidad Complutense de Madrid \\ Madrid, Spain \\ hidalgo@dacya.ucm.es
}

\author{
J. Manuel Colmenar \\ Universidad Rey Juan Carlos \\ Móstoles, Spain \\ josemanuel.colmenar@urjc.es \\ Sancho Salcedo-Sanz \\ Universidad de Alcalá \\ Alcalá de Henares, Spain \\ sancho.salcedo@uah.es
}

\begin{abstract}
Energy is essential for all countries, since it is in the core of social and economic development. Since the industrial revolution, the demand for energy has increased exponentially. It is expected that the energy consumption in the world increases by $50 \%$ by 2030 [17]. As such, managing the demand of energy is of the uttermost importance. The development of tools to model and accurately predict the demand of energy is very important to policy makers. In this paper we propose the use of the Structured Grammatical Evolution (SGE) algorithm to evolve models of energy demand, over macro-economic indicators. The proposed SGE is hybridised with a Differential Evolution approach in order to obtain the parameters of the models evolved which better fit the real energy demand. We have tested the performance of the proposed approach in a problem of total energy demand estimation in Spain, where we show that the SGE is able to generate extremely accurate and robust models for the energy prediction within one year time-horizon.
\end{abstract}

\section{CCS CONCEPTS}

- Computing methodologies $\rightarrow$ Artificial intelligence; Model development and analysis; $\bullet$ Theory of computation $\rightarrow$ Algorithm design techniques;

\section{KEYWORDS}

Grammatical Evolution, Structured Grammatical Evolution, Performance.

\section{ACM Reference Format:}

Nuno Lourenço, J. Manuel Colmenar, J. Ignacio Hidalgo, and Sancho SalcedoSanz. 2020. Evolving Energy Demand Estimation Models over Macroeconomic Indicators. In Genetic and Evolutionary Computation Conference (GECCO '20), July 8-12, 2020, CancÃžn, Mexico. ACM, New York, NY, USA, 7 pages. https://doi.org/10.1145/3377930.3390153

Permission to make digital or hard copies of all or part of this work for personal or classroom use is granted without fee provided that copies are not made or distributed for profit or commercial advantage and that copies bear this notice and the full citation on the first page. Copyrights for components of this work owned by others than ACM must be honored. Abstracting with credit is permitted. To copy otherwise, or republish, to post on servers or to redistribute to lists, requires prior specific permission and/or a fee. Request permissions from permissions@acm.org.

GECCO '20, July 8-12, 2020, CancÃžn, Mexico

(C) 2020 Association for Computing Machinery.

ACM ISBN 978-1-4503-7128-5/20/07 ..\$15.00

https://doi.org/10.1145/3377930.3390153

\section{INTRODUCTION}

The estimation of the total energy demand of a country based on macro-economic variables is an important problem for policy makers [17], with hard economical, environmental and social implications. It is known that energy demand is one of the important economic indicators of growth for a country [13], as it has been proven that energy demand reduces during economic crises. On the contrary, the demand for energy soars in periods of healthy economic behaviour of a country [7], mainly pushed up by energydemanding sectors such as industry and construction [1].

Usually, the energy demand estimation problem has been tackled using one year-ahead prediction time horizon, though other longer-term horizon estimation are possible as well. There are a good number of previous works on this problem in the literature: the first work dealing with energy demand from macro-economic indicators was [1], where a Genetic Algorithm (GA) was proposed to obtain the parameters of an exponential prediction model for energy demand in Turkey. There have also been works dealing with Particle Swarm Optimization (PSO) in different versions of this problem, such as [18] for energy demand or [6] for electricity demand estimation in Turkey. Hybrid algorithms involving PSO and GAs have been proposed in [19-21] applied to problems of energy demand estimation in China. In [5], a neural network was applied to solve a problem of total energy demand estimation in Korea. A similar approach based on neural networks for the case of energy demand estimation in Greece has been presented in [3]. More recently, in [14] a Harmony Search approach with feature selection has been proposed for a problem of energy demand estimation in Spain, and in [15] a Variable Neighbourhood Search (VNS) approach was employed for solving this problem, also comparing the results with that of an Extreme Learning Machine (ELM). In [2] new models for energy demand estimation were proposed based on a Grammatical Evolution (GE) approach combined with a Differential Evolution (DE) algorithm. After this paper, a Grammatical Swarm (GS) method was also hybridized with DE in [11], improving the results of the previous work.

In this paper we propose a Structured Grammatical Evolution algorithm (SGE) hybridized with DE for tackling a problem of energy demand estimation in Spain. Specifically, the SGE module proposes different models for energy demand, whereas the DE optimizes the parameters of these models according to the real data (energy demand and input macro-economic variables) of the problem. The 
main advantage of SGE in relation to GE is the reduction of the redundancy in the representation of individuals, as well as the increasing of the locality in the space of solutions [10]. These features make SGE more effective and robust in the exploration of solutions than GE [8], being able to obtain even better results than the GS proposal.

The rest of this paper is structured as follows: Section 2 describes how the proposed SGE is able to generate efficient demand estimation models for energy. Section 3 shows the specific adaptation of the SGE grammars and the DE proposed to obtain the final estimation of the energy demand from macro-economic indicators. Section 4 presents the experimental set of the paper, where macro-economic data and the energy demand estimation of Spain is tackled. Finally, Section 5 closes the paper by giving some concluding remarks on the work carried out.

\section{MODEL GENERATION WITH SGE}

Structured Grammatical Evolution (SGE) $[9,10]$ is a recently proposed variant of GE which solves some of its main drawbacks. The main characteristic of SGE is that it uses a one-to-one mapping between each gene and a non-terminal symbol of the grammar. This arrangement of the genotype ensures that when a change occurs in one of the genes, it does not affect the derivation options of other non-terminals, thus restricting the modifications that can occur at phenotypic level (i.e., the derivation tree). This way, we can increase the locality of the representation. Additionally, the values inside each list are bounded by the number of expansion options of the corresponding non-terminal. Consequently, the modulus operator is not needed, which decreases the redundancy introduced by it.

Differential Evolution (DE) is an evolutionary algorithm, presented in [16], which has been proven to be very useful in parameter optimization [12]. The individuals of the population are represented as vectors of real numbers. Hence, a direct decoding can be made, making each element of the vector correspond to a parameter of the optimized element.

Therefore, the proposed model generation follows the same idea as in [2], where a GE algorithm is hybridized with a DE metaheuristic to produce new models for energy demand. In this case, the SGE is combined with DE to produce the optimized models for energy demand.

As shown in Figure 1, the main process receives a set of parameters for both SGE and DE algorithms, which consists of the number of generations, population size, etc., that will be detailed later in this paper. On each generation, the SGE process, guided by the grammar definition, generates a population of parameterized models. These models are taken by the DE process which, for each model, parses the given expression and automatically configures the number of variables according to the number of different parameters included in the model.

For example, a population of 3 parameterized models could be the following:

$$
\begin{gathered}
w_{1} \times x_{1}+\left(x_{2} \times w_{2}\right)-x_{3} \\
w_{0}+\left(w_{1} / x_{1}\right)+\left(w_{3} \times x_{3}\right) \\
w_{0}-\left(w_{1} / x_{1}\right)+\left(x_{2} \times w_{2}\right)-w_{3} \times x_{3}
\end{gathered}
$$

In this toy example the parameters are represented by the $w_{i}$ elements and the variables by the $x_{i}$ elements. As seen, the first two models have 3 parameters and 2 variables, while the third one has 4 parameters and 3 variables.

Then, the DE process is run for each model, taking into account the training data, and obtaining the best parameter values for each one. Hence, the fitness values of the models $\left(f_{1}, f_{2}, \cdots, f_{N}\right.$ in Figure 1) are returned in order to continue the evolutionary process of SGE. Finally, the best model and its corresponding parameter values are returned by the hybrid algorithm.

As it can be inferred from the figure, this a generic process of model generation that can be adapted to any different context by selecting the appropriate grammar and training data. Besides, the parameter values for both the SGE and the DE processes must be accordingly tuned. In the next section, we will describe how this generic process has been adapted to the energy prediction scenario.

\section{ENERGY PREDICTION}

As stated in the previous section, the process of energy prediction mainly depends on the grammar of SGE, which is in charge of finding the structure of the models, and the training data, which will allow the DE obtaining the best parameter configuration.

In this work, we have adapted the grammars proposed in [11], defined for the classical GE approach, to the SGE particular case. More precisely, we have used the grammar shown in Figure 2 for the experimental experience. As it can be seen, the grammar is formed by six derivation rules corresponding to the start symbol $(<$ star $\mathrm{t}\rangle$ ), the two non-terminal symbols $(<$ recExpr $>$ and $<$ expr $>$ ), and the three terminals ( $<$ param $>,<$ var $>$ and $<o p>)$.

The grammar does not strictly fix the structure of the model, because the $<r e c E x p r>$ element allows the extension of the mathematical expression. Besides, there is no constraint about the arithmetic operators. Therefore, the aim of this grammar is to let the SGE algorithm select the best model structure, that is, choose and operate those variables that better contribute to the model of the target value. However, the grammar forces each variable to be always operated with, at least, one parameter. This way, the weight of each one of the selected variables will be adjusted by the DE algorithm, performing an intensification process for each candidate model.

Since the grammar has six derivation rules, each SGE individual will have six genes, which will be linked to a specific symbol. To calculate the length of the lists inside each gene, the algorithm computes the maximum number of derivation options for each element. Table 1 gives an example of the genotype of an individual created using the grammar of Figure 2, and how we derive it into a predictive model for energy demand estimation. As it can be seen in the table, the column labelled as Integers left shows six lists of integers, which are the genes, in the same order as the derivation rules of the grammar. Hence, the first gene corresponds to the <start $>$ symbol and, given that has only one production rule, the value of the gene is processed substituting it by the $\mathrm{w}[0]<\mathrm{op}>\langle\mathrm{recExpr}>$ expression in the second row. Then, the $<o p>$ symbol has to be decoded. The corresponding gene is the sixth one, because this symbol is decoded by rule VI in the grammar. As it can be seen in the table, the value that is used for decoding is 0 , which translates the <op> symbol 


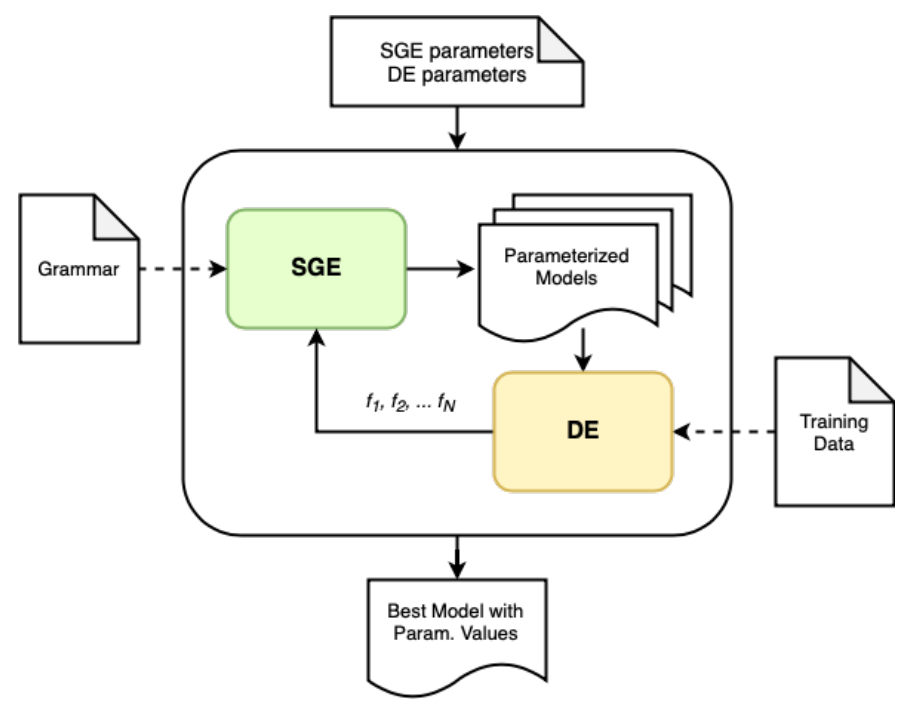

Figure 1: Flow diagram with the algorithmic SGE+DE process.

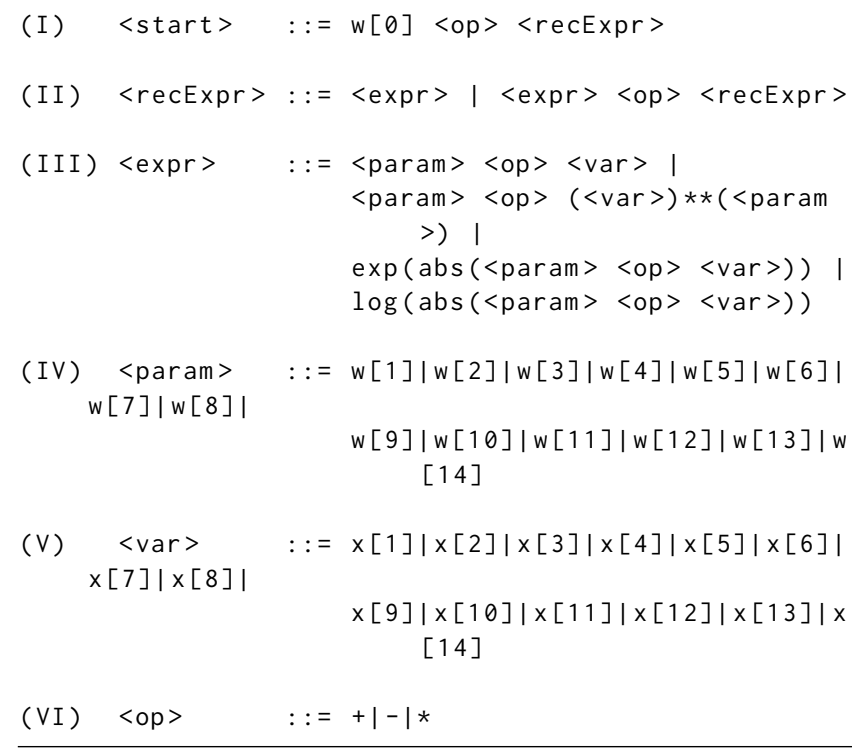

Figure 2: Grammar used for SGE experiments, adapted from [11].

into $\mathrm{a}+$. The decoding process continues till all the non-terminals are processed, returning the expression that is shown in the last row of the table.

\section{EXPERIMENTAL RESULTS}

In this section we show the ability of the proposed algorithm to produce models which use macro-economic inputs to predict the energy demanded in Spain, with a prediction time-horizon of one year.
Table 1: Mapping procedure that converts an individual (first row, right column) into a polynomial expression using SGE. Each row represents a derivation step using the grammar in Figure 2. Each gene has the list of integers needed for expanding $<$ start $>$, $<$ recExpr $>$, $<$ expr $>$, $<$ param $>$, $<$ var $>$ and $<$ op $>$, respectively. Adapted from [9].

\begin{tabular}{|c|c|}
\hline Derivation step & Integers left \\
\hline$<$ start $>$ & {$[[0],[1,0],[0,0],[8,5],[11,4],[0,2,1,2]]$} \\
\hline$w[0]<$ op $><$ recExpr $>$ & []$],[1,0],[0,0],[8,5],[11,4],[0,2,1,2]]$ \\
\hline$w[0]+<$ recExpr $>$ & {$[[],[1,0],[0,0],[8,5],[11,4],[2,1,2]]$} \\
\hline$w[0]+<\operatorname{expr}><\mathrm{op}><$ recExpr $>$ & {$[[],[0],[0],[8,5],[11,4],[2,1,2]]$} \\
\hline$w[0]+<$ param $><$ op $><$ var $><$ op $><$ recExpr $>$ & {$[[],[0],[0],[8,5],[11,4],[2,1,2]]$} \\
\hline$w[0]+w[10]<$ op $><$ var $><$ op $><$ recExpr $>$ & {$[[],[0],[0],[5],[11,4],[2,1,2]]$} \\
\hline$w[0]+w[10] *<\operatorname{var}><$ op $><$ recExpr $>$ & {$[[],[0],[0],[5],[11,4],[1,2]]$} \\
\hline$w[0]+w[10] * x[13]<\mathrm{op}><\mathrm{recExpr}>$ & {$[[],[0],[0],[5],[4],[1,2]]$} \\
\hline$w[0]+w[10] * x[13]-<\operatorname{expr}>$ & {$[[],[],[0],[5],[4],[,, 2]]$} \\
\hline$w[0]+w[10] * x[13]-<$ param $><$ op $><$ var $>$ & {$[[],[],[],[5],[4],[2]]$} \\
\hline$w[0]+w[10] * x[13]-w[6]<\mathrm{op}><\operatorname{var}>$ & {$[[],[],[],[],[4],[2]]$} \\
\hline$w[0]+v$ & {$[[],[],[],[],[4],[]]$} \\
\hline$w[0]+w[$ & {$[[],[],[],[],[],[]]$} \\
\hline
\end{tabular}

\subsection{Setup}

In order to make a fair comparison, we use the same numerical parameters described in the literature whenever is possible [11]. For the SGE algorithm we defined a population of 50 individuals that are evaluated throughout 40 generations. We allow $10 \%$ of the best individuals to survive untouched and pass along to the next generation without any changes. The probabilities of applying the variation operators are 0.65 for recombination and 0.02 for mutation. Regarding the parameters related to the genotypic representation, we have a genotype that allows derivation trees with depth varying between 5 and 17. On the other hand, in the configuration of the DE method we have used a population of 75 individuals, which are evolved during 100 generations. For the rate of the variation operators we used 0.8803 for crossover and 0.4717 for mutation [2].

Each algorithm is executed 30 times in order to allow the statistical analysis. Table 2 summarises the parameters of each algorithm. 
Table 2: Parameters used in the experimental analysis for each method.

\begin{tabular}{lc}
\hline Parameter & GE \\
\hline Number of runs & 30 \\
Population Size & 50 \\
Generations & 40 \\
Selection Method & Tournament with size 3 \\
Elitism & $10 \%$ \\
Crossover Rate & 0.65 \\
Mutation Rate & 0.02 \\
Initialisation & Random \\
Min. Initialisation Depth & 5 \\
Max. Initialisation Depth & 6 \\
Max. Tree Depth & 17 \\
\hline & \\
\hline Population Size & 75 \\
Generations & 100 \\
DE Strategy & DE \\
Crossover Rate (CR) & 0.8803 \\
Mutation Rate (Differential Weight) & 0.4717 \\
\hline
\end{tabular}

The evolutionary process is guided by the minimization of the sum of the absolute errors between the original data and the predictions by the models being evolved. This fitness function, denoted as SAE, is defined in Eq. (1), where $T$ is the number of data points, $\hat{y}_{i}$ is the predicted value for sample $i$, and $y_{i}$ is the reference value for sample $i$.

$$
\mathrm{SAE}=\sum_{i=1}^{T}\left(\left|\hat{y}_{i}-y_{i}\right|\right)
$$

\subsection{Dataset for Energy Demand Estimation}

The dataset used for our experiments correspond to real data of Spain, and it is composed by the following 14 macro-economic variables:

(1) Gross Domestic Product $(€)$

(2) Population

(3) Exports $(€)$

(4) Imports $(€)$

(5) Energy production (kTOE)

(6) Electricity power transport $(\mathrm{kWh})$

(7) Electricity production (kWh)

(8) GDP per unit of energy use (e per kTOE)

(9) Energy imports net (\% use)

(10) Fossil fuel consumption (\% total)

(11) Electric power consumption (kWh)

(12) CO2 emissions (Mton)

(13) Unemployment rate

(14) Diesel consumption in road (kTOE)

All the variables have been gathered between the years of 1980 and 2011. The dataset is divided into two parts, one for training and one for test. The training set is formed by the following 15 years: 1983, 1985, 1987, 1988, 1990, 1991, 1993, 1995, 1999, 2002, 2004, 2007,
$2009,2010,2011$. The test set is composed by the following 16 years: 1982, 1984, 1986, 1989, 1992, 1994, 1996, 1997, 1998, 2000, 2001, 2003, $2005,2006,2008$. All the data was standardised acording the to min and max values.

\subsection{Training Results}

The experiments in this section aim to gain insights on the ability of the SGE+DE algorithm to evolve models that can accurately predict the energy required in a given year. As stated before, we consider the minimization of the sum of absolute errors between the observed data and the predictions resulting from applying the models obtained during the search process. Each model receives as input the features observed in year $t$ and return an estimation of energy for the year $t+1$. The results are presented in terms of mean best fitness (MBF) averaged over 30 runs.

Figure 3 shows the evolution of MBF across the 40 generations, averaging the 30 runs. An overview of the results shows that the error of the evolved models steadily decreases over the entire search process. Looking at the performance of the method, it is possible to see a steep descent in the error in the first 15 generations. After this point, the error continues to decrease but at a slower rate. The evolved methods do not reach a mean error of 0 in the time assigned to the optimisation, but the trend of the fitness suggests that if we extended the evolutionary process for a few more generations, it would be enough to reach solutions with error 0 .

In Table 3 we can see a summary of the runs for the energy demand prediction obtained in the training data. We can see that the MBF is close to zero, and more importantly, that the standard deviation is very small. This is an important result, since it tells us that SGE is evolving models that can accurately predict the demand of energy, but also that the method is robust, in the sense that, for different runs, the models have very similar predictions.

Table 3: Best and mean best fitness results of the energy prediction estimations for the training dataset in Spain. Values closer to zero are better.

\begin{tabular}{c|c}
\multicolumn{2}{c}{ Training } \\
\hline Best Fitness & Mean Best Fitness \\
\hline 0.09 & $0.20( \pm 0.09)$ \\
\hline
\end{tabular}

Finally, the best model evolved is presented in Eq. (2). The $x_{1}, \cdots, 14$ symbols correspond to the macro-economic variables defined above, and $w_{0, \cdots, 14}$ correspond to the weights that are further optimised by the DE algorithm. The predictions of this model for the training data can be seen in Figure 4, where the target value is presented in the normalized form. The solid line corresponds to the observed and the dashed line corresponds to the predict data. As one can see, both lines overlap for most of the years. There is however a small difference between 2004 and 2007 where the evolved model underestimates the demand of energy.

$$
\begin{gathered}
w_{0}+w_{6} * x_{1} * \log \left(\operatorname{abs}\left(w_{2} * x_{1}\right)\right)+w_{9}-\left(x_{11}\right)^{\left(w_{11}\right)}-w_{10} *\left(x_{12}\right)^{\left(w_{3}\right)}- \\
\exp \left(\operatorname{abs}\left(w_{13} * x_{11}\right)\right) * w_{13}-x_{1} * w_{11}-x_{8} * w_{14}-\left(x_{4}\right)^{\left(w_{5}\right)}-w_{1} *\left(x_{6}\right)^{\left(w_{13}\right)}
\end{gathered}
$$




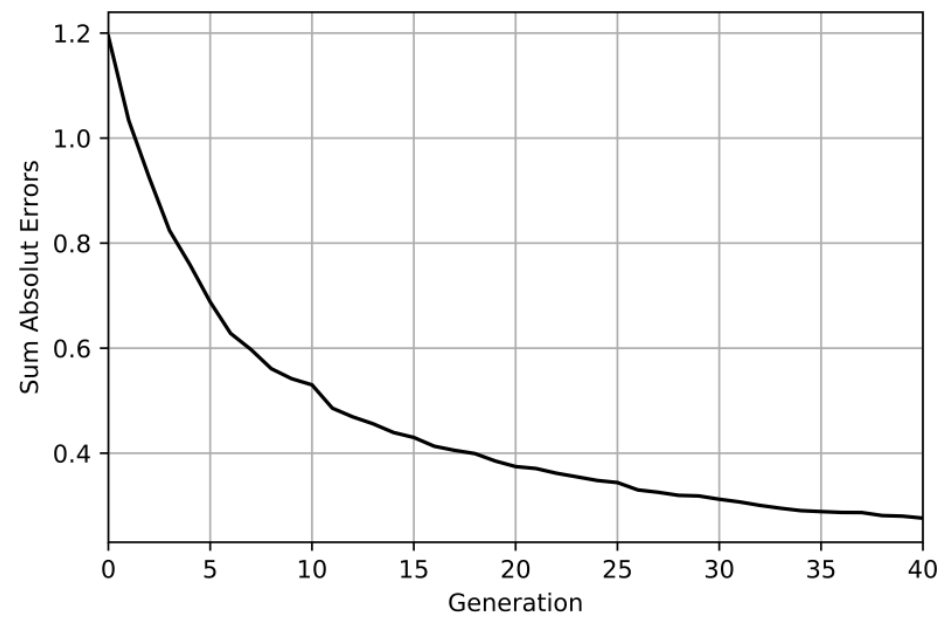

Figure 3: Mean Best Fitness evolution along the 40 generations. The results are averages of 30 runs.

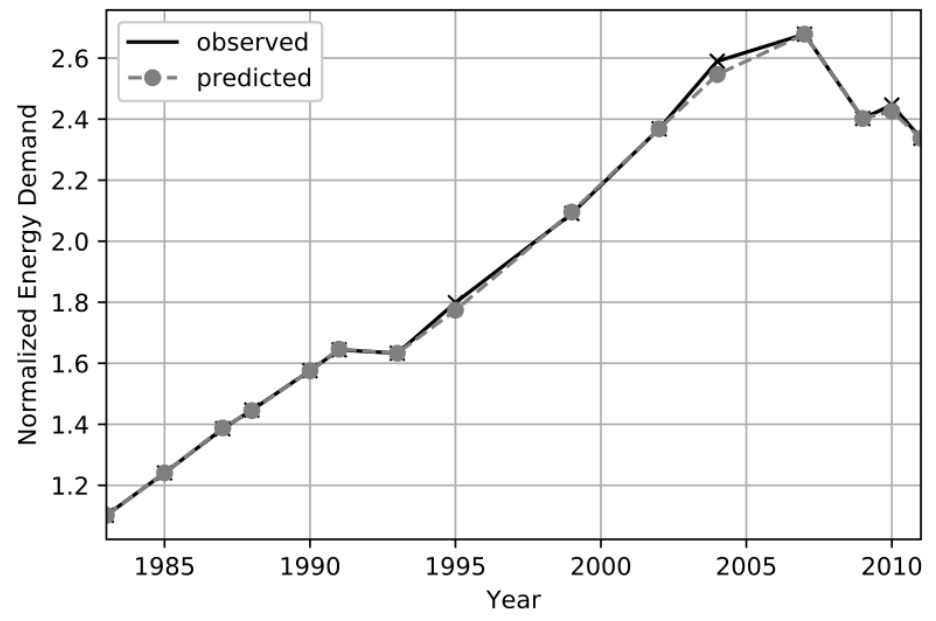

Figure 4: Predictions of the best evolved model (Eq. (2)) for the training data.

\subsection{Testing Results}

In this section we analyse the generalisation ability of the models evolved by the $\mathrm{SGE}+\mathrm{DE}$ algorithm. We start by analysing the results of the predictions for data that were not used for training the algorithm. Then, we compare the results obtained with the ones from the GS approach presented in [11]. To compare the approaches we use the Mann-Whitney test due to the fact that the data do not follow a normal distribution, and the initial populations are different for both algorithms. We use a significance level $\alpha=0.05$. When statistical differences are found, we compute the effect size to assess how meaningful the differences are. The effect size can be low $(0.1$ $\leq \mathrm{r}<0.3)$, medium $(0.3 \leq \mathrm{r}<0.5)$ or large $(\mathrm{r} \geq 0.5)[4]$.
A summary of the obtained results are presented in Table 4. Looking at the results, it is possible to see that they follow the trend presented in training, i.e., the models have a low error concerning the predictions of the energy demand. These results indicate that the models generated by SGE are not overfitting the training data, and are capable of representing the real patterns of the data. Another interesting aspect is that the standard deviation of the evolved models is still quite small, which is an indication of their robustness.

Regarding the comparison with the GS approach it is possible to see that the results obtained by SGE, in terms of error, are considerably smaller than those by the GS approach (Table 4). Not only the SGE attains a smaller error, but it also is able to have a smaller standard deviation (Figure 5). 


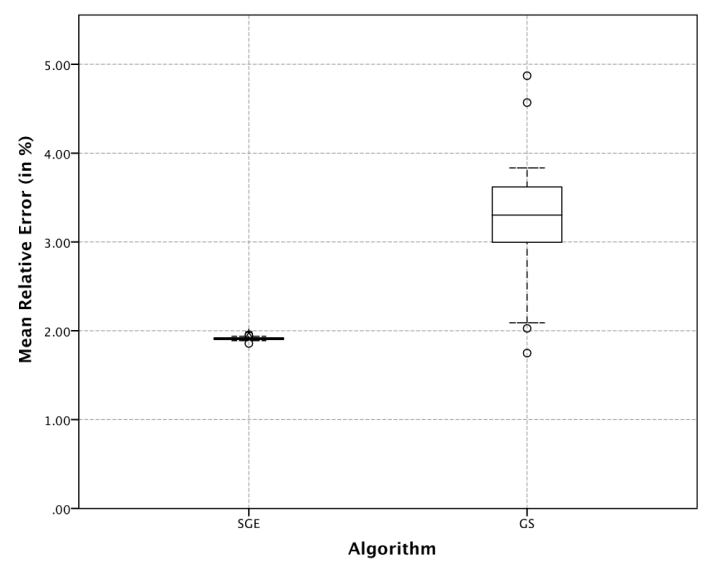

Figure 5: Comparison of the prediction results obtained with SGE and GS in the Test data. Values close to zero are better.

To further confirm the effectiveness of the the SGE approach, we employed a sound statistical analysis, and found that the models evolved SGE are statistically significant different from the GS ones (p-value $<0.000)$ and the effect size is large $(r>0.5)$.

Finally, Figure 6 shows the predictions made by the best model evolved and the actual observations in the test set using the real units for energy demand (kTOE). As we can see, the results follow the same pattern observed in the training data, where the SGE model tends to underestimate the demand of energy in some years, even though the trends that the data follows are captured by the model. These results confirm the quality of the evolved models, and the robustness of the proposed method.

Table 4: Best and Mean Best Fitness results of the energy prediction estimations for the testing dataset in Spain. Values closer to zero are better. The ${ }^{* * *}$ indicate that the algorithm has statistical significant differences, and that the effect size is large

\begin{tabular}{c|c|c}
\multicolumn{3}{c}{ Testing } \\
\hline Algorithm & Best Fitness & Mean Best Fitness \\
\hline SGE & $\mathbf{1 . 1 8}^{* * *}$ & $\mathbf{1 . 9 1 (} \pm \mathbf{0 . 0 0 4})$ \\
GS & 1.75 & $3.27( \pm 0.124)$ \\
\hline
\end{tabular}

\section{CONCLUSIONS AND FUTURE WORK}

Since the dawn of industrial revolution, the demand for energy has greatly increased, and it has become essential in all countries, since it provides sustainable social, economic and/or environmental developments. Managing energy demand is of the uttermost importance for policy makers, and the development of tools to model and accurately predict the demand is crucial for new energetic policies.

In this work we investigate the ability of Structured Grammatical Evolution (SGE) to search for predictive models for total energy demand in Spain. SGE represents the individuals as chromosomes where each gene is composed of a list of values corresponding to the productions of each non-terminal symbol. This way, the decoding

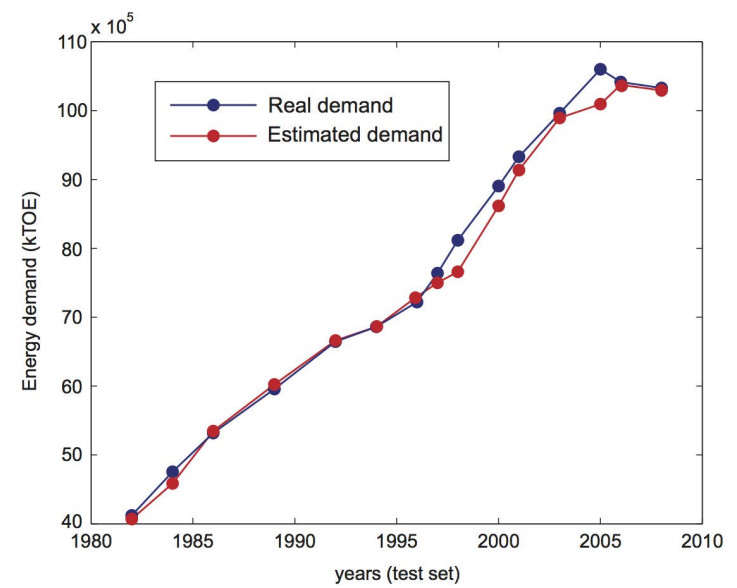

Figure 6: Predictions of the best evolved model for the testing data between 1982 and 2008.

process is different to GE and can increase the locality of the genetic operators. These particularities of SGE make it more robust and make it ideal to be combined with DE approach.

The experimental results conducted reveal that the majority of the 30 runs of the proposed approach produced models better than previous approaches. Another interesting result, is concerned with the very robustness of SGE, since the best models produced have very low standard deviation between the different runs. The proposed approach shows its ability to avoid the drawbacks of GE, obtaining excellent predictions of total energy demand in Spain with one year time-horizon.

Moreover, we compared the obtained results with the ones from the a Grammatical Swarm (GS) [11], which is one of the most effective approaches to the problem at hand. The comparison shows that not only SGE obtains models that are better (i.e, they have a smaller error), but also they are more robust, since they have a significant smaller standard deviation.

In future work we will consider the study of other countries energy demand with this methodology, and the inclusion of a different set of macro-economic indicators or alternative inputs which can be related to the energy consumption in a given country.

\section{ACKNOWLEDGEMENTS}

This work has been supported by Fundación Eugenio Rodriguez Pascual 2019-20 grant, Spanish Ministerio de Ciencia, Innovación y Universidades (MCIU/AEI/FEDER, UE) under grant refs. RTI2018095180-B-I00 and PGC2018-095322-B-C22, Spanish Ministerio de Economía y Competitividad, grant number TIN2017-85887-C2-2P; Madrid Regional Goverment - FEDER grants B2017/BMD3773 (GenObIA-CM), Y2018/NMT-4668 (Micro-Stress- MAP-CM) and P2018/TCS-4566 (CYNAMON).

This work was also supported by national funds through the FCT - Foundation for Science and Technology, I.P., within the scope of the project CISUC - UID/CEC/00326/2020 and by European Social Fund, through the Regional Operational Program Centro 2020. 


\section{REFERENCES}

[1] H. Ceylan and H. K. Ozturk. 2004. Estimating energy demand of Turkey based on economic indicators using genetic algorithm approach. Energy Conversion and Management 45 (2004), 2525 - 2537.

[2] J.M. Colmenar, J.I. Hidalgo, and S. Salcedo-Sanz. 2018. Automatic generation of models for energy demand estimation using Grammatical Evolution. Energy 164 (2018), $183-193$

[3] L. Ekonomou. 2010. Greek long-term energy consumption prediction using artificial neural networks. Energy 35, 2 (2010), 512 - 517.

[4] Andy Field. 2009. Discovering statistics using SPSS. Sage publications.

[5] Z. W. Geem and W. E. Roper. 2009. Energy demand estimation of South Korea using artificial neural network. Energy Policy 37 (2009), 4049-4054.

[6] M. S. Kiran, E. Özceylan, M. Gündüz, and T. Paksoy. 2012. Swarm intelligence approaches to estimate electricity energy demand in Turkey. Knowledge-Based Systems 36 (2012), 93-103.

[7] Fangyi Li, Zhouying Song, and Weidong Liu. 2014. China's energy consumption under the global economic crisis: Decomposition and sectoral analysis. Energy Policy 64 (2014), 193 - 202.

[8] Nuno Lourenço, J. Manuel Colmenar, J. Ignacio Hidalgo, and Óscar Garnica. 2019 Structured Grammatical Evolution for Glucose Prediction in Diabetic Patients. In Proceedings of the Genetic and Evolutionary Computation Conference (GECCO '19). ACM, New York, NY, USA, 1250-1257.

[9] Nuno Lourenço, Filipe Assunção, Francisco B Pereira, Ernesto Costa, and Penousal Machado. 2018. Structured grammatical evolution: a dynamic approach. In Handbook of Grammatical Evolution. Springer, 137-161.

[10] Nuno Lourenço, Francisco B Pereira, and Ernesto Costa. 2016. Unveiling the properties of structured grammatical evolution. Genetic Programming and Evolvable Machines 17, 3 (2016), 251-289.

[11] David Martínez-Rodríguez, J Manuel Colmenar, J Ignacio Hidalgo, Rafael-J Villanueva Micó, and Sancho Salcedo-Sanz. 2019. Particle swarm grammatical evolution for energy demand estimation. Energy Science \& Engineering (2019).

[12] Kenneth V Price. 2013. Differential evolution. In Handbook of Optimization. Springer, $187-214$.

[13] Karolina Safarzynska and Jeroen C. J. M. van den Bergh. 2017. Integrated crisisenergy policy: Macro-evolutionary modelling of technology, finance and energy interactions. Technological Forecasting and Social Change 114 (2017), 119 - 137.

[14] S. Salcedo-Sanz, J. Muñoz-Bulnes, J.A. Portilla-Figueras, and J. Del Ser. 2015. One-year-ahead energy demand estimation from macroeconomic variables using computational intelligence algorithms. Energy Conversion and Management 99 (2015), $62-71$.

[15] J. Sánchez-Oro, A. Duarte, and S. Salcedo-Sanz. 2016. Robust total energy demand estimation with a hybrid Variable Neighborhood Search âĂŞ Extreme Learning Machine algorithm. Energy Conversion and Management 123 (2016), 445 - 452.

[16] Rainer Storn and Kenneth Price. 1997. Differential Evolution - A Simple and Efficient Heuristic for global Optimization over Continuous Spaces. Fournal of Global Optimization 11, 4 (01 Dec 1997), 341-359.

[17] L. Suganthi and A. A. Samuel. 2012. Energy models for demand forecasting-A review. Renewable and Sustainable Energy Reviews 16 (2012), 1223-1240.

[18] A. Unler. 2008. Improvement of energy demand forecasts using swarm intelligence: The case of Turkey with projections to 2025. Energy Policy 36 (2008), 1937-1944.

[19] S. Yu, Y. M. Wei, and K. Wang. 2012. A PSO-GA optimal model to estimate primary energy demand of China. Energy Policy 42 (2012), 329-340.

[20] S. Yu, K. Zhu, and X. Zhang. 2012. Energy demand projection of China using a path-coefficient analysis and PSO-GA approach. Energy Conversion and Management 53, 1 (2012), 142-153.

[21] S. Yu and K. J. Zhu. 2012. A hybrid procedure for energy demand forecasting in China. Energy 37 (2012), 396-404. 